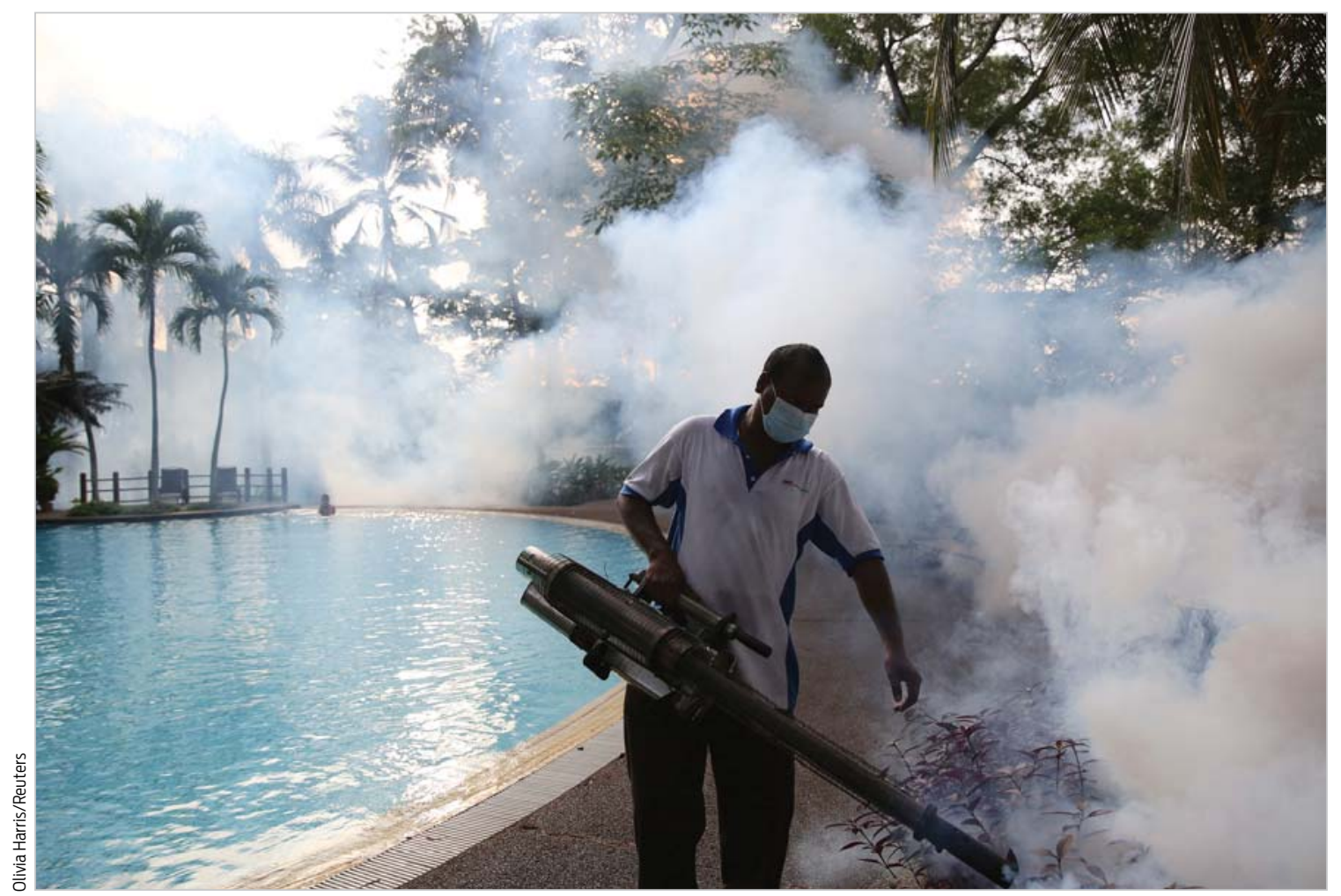

A pest control worker sprays insecticide to help control the spread of dengue fever carried by mosquitoes in Kuala Lumpur. This year, Malaysia has seen a $200 \%$ increase in cases of dengue fever compared to the same period in 2013, local media reported.

\title{
Research translation and chronic illness
}

The Australian Institute of Health and Welfare estimates that over 7 million Australians are living with at least one chronic condition, and many of these are living with more than one (http://www.aihw.gov.au/chronicdiseases). This places a heavy human and financial burden on the shoulders of the individuals with these conditions, their families and carers, and the health care system. Research that can relieve these burdens and improve health outcomes is vital but only useful if it is translated appropriately.

Now in its third year, almost 3000 NHMRC investigators have signed up for the Research Translation Faculty. As it slowly matures, and the Steering Groups get closer to completing their first Cases for Action (http://www. nhmrc.gov.au/research-translation/ research-translation-faculty/research- translation-faculty-steering-groups), we now look forward to the key event on the Faculty's agenda - the annual symposium. The focus this year will be on how, through more effective research translation, we might achieve better health outcomes for the millions of Australians living with chronic conditions. Some of Australia's foremost health and medical researchers will talk about their work in translating research on a range of chronic conditions and health care settings, and discuss some of the successes and challenges along the way.

Special attention will be paid to the health system issues that make translating research in chronic disease into decision making in rural and remote settings particularly challenging. Also to be addressed is how research translation can

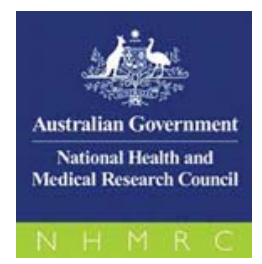

positively influence ageing and health, which is particularly relevant as the rates of chronic illness increase with age.

For research to be relevant, conversations cannot be unidirectional, where the researcher pushes their results at the decisionmaker, or vice versa. The second day of the symposium will therefore start by investigating optimal strategies for designing research that will have an impact, and will end with a panel discussion on the challenges of making health care decisions when bombarded with health care information and conflicting pieces of research.

Anyone with an interest in research translation is welcome to attend. More information can be found at http://

doi: 10.5694/mjal4.01133 


\section{Three devices with promise}

Medical News Today reports on three new medical devices which are showing promise in trials. The US Food and Drug Administration has granted clearance for the mobile-based app AliveCor Heart Monitor, which can detect atrial fibrillation through real-time electrocardiogram recordings. The Oculus Rift virtual reality headset is being tested by scientists, videographers and surgeons as a potential training aid for surgeons in a "low- to no-risk simulated environment" that will not be prohibitively expensive. The University of Leicester in the UK has developed a "rapid and sensitive electronic nose" that can sniff out the highly infectious hospital superbug Clostridium difficile. The team of chemists and a microbiologist claim that the device can detect not only the bacterium, but also the specific strain.

\section{Ebola drug trials set to begin}

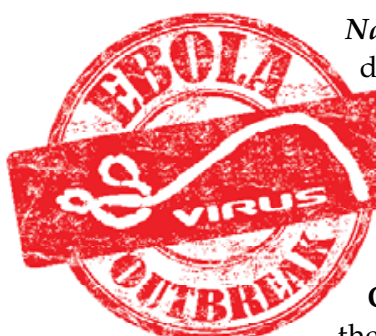

Nature reports that experimental Ebola drug ZMapp is set to be tested in clinical trials on humans after being used on seven people so far, two of whom did not survive, on a caseby-case basis. An expert panel convened by the World Health Organization last month decided that the exceptional impact, both socially and economically, of the current Ebola outbreak warranted the use of unapproved drugs and vaccines. On 4 and 5 September, 150 Ebola scientists, pharma executives, clinical trials experts, ethicists and regulatory officials met to prioritise the most promising products for use in clinical trials.

\section{Dengue fever outbreak in Japan}

For the first time in almost 70 years, Japan is battling an outbreak of dengue fever with at least 34 confirmed cases early

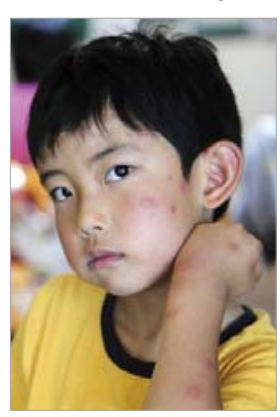
in September, the ABC reports. Tokyo's Yoyogi Park, the city's largest open space, is believed to be ground zero for the mosquito-borne disease. None of the cases are life-threatening, according to authorities. The park's ponds have been drained and sprayed with insecticide. Japan has had an exceptionally wet summer that may have contributed to the outbreak, which is thought to have originated with a returning traveller from South-East Asia.

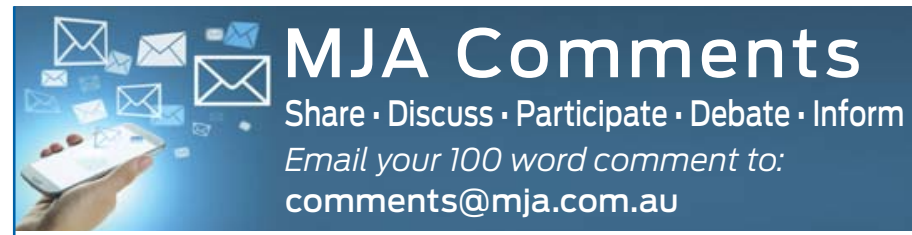

Send us your comments on this issue's suggested topic:

\section{How can we best balance the professional freedom of doctors with concern for patient safety? Are we handling impaired doctors optimally?}

\section{Be heard!}

Why must questioners at public and, especially, medical meetings grasp the proffered mobile microphone as if it were covered with cocci causing them to hold it many inches from their mouths?

Why must they loll instead of standing, address the lectern continuously whilst paying no heed to the audience around them and at their rear who are straining fruitlessly to hear and understand, even perhaps eager to participate in the exchange?

In this setting the question becomes a soliloquy rather than an audible and intelligible declamation.

A prominent sign at the front of the lecture theatre at Sir Charles Gairdner Hospital reads: "Be heard! Your questions and views enlighten us all".

Alex Cohen

Retired Professor, Perth, WA

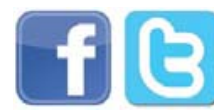

Follow us on Twitter (@theMJA) and like us on Facebook (https://www.facebook.com/theMJA)

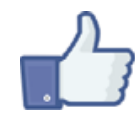

Email us a brief comment (no more than 100 words) on any health-related subject. Email your comments to: comments@mja.com.au When sending your comment, please include your full name, discipline and state of residence, and disclose any relevant information or affiliations that may affect interpretation of your comments. Visit: https://www.mja.com.au/journal/ mja-instructions-authors-types-articles-published-mja\#Comments

\section{MJA InSight Poll}

Should medical researchers declare personal as well as financial influences on their research?

Total respondents: 70

Take part in next week's poll on: www.mja.com.au/insight

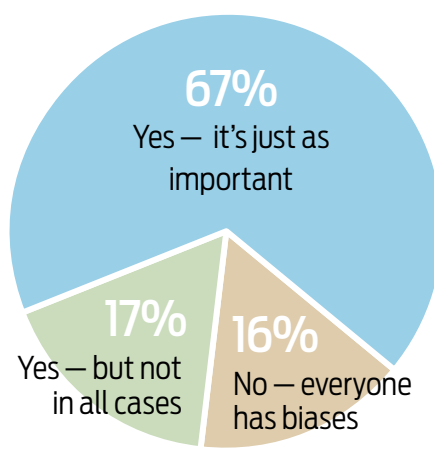

No- everyone
has biases

\section{NHMRC awards 17 Practitioner Fellowships}

The National Health and Medical Research Council has awarded 17 grants to Australia's best clinical researchers to "conduct research that is strongly informed by their careers in health care". Awardees included Professor Katrina Allen (\$459 687) from the Murdoch Childrens Research Institute, for food allergy prevention; Professor Jodie Dodd (\$551 436) from the University of Adelaide, for research on maternal overweight and obesity; and Professor Jon Emery (\$459 687) from the University of Melbourne, for work on earlier cancer diagnosis. The other Fellowships were awarded to Associate Professor Louise Maple-Brown (Menzies School of Health Research), Associate Professor Jane Pillow (University of Western Australia), Professor Paul Fitzgerald (Monash University), Professor Stephen Fox (University of Melbourne), Professor Rachelle Buchbinder (Monash University), Professor Vicki Anderson (Murdoch Childrens Research Institute), Professor David Hunter (University of Sydney), Professor R Douglas McEvoy (Flinders University), Associate Professor Helmut Butzkueven (University of Melbourne), Professor Helen Herrman (University of Melbourne), Professor John Myburgh (University of New South Wales), Associate Professor Yves d'Udekem d'Acoz (Murdoch Childrens Research Institute), Professor Ben Mol (University of Adelaide), and Professor Andrew Scott (Ludwig Institute for Cancer Research). 